\title{
On the estimation of hospital beds occupancy after hip surgery
}

\author{
S. D. Sousa ${ }^{1}$, C. S. Rodrigues ${ }^{1}$, E. P. Nunes ${ }^{1}$ \\ ${ }^{1}$ ALGORITMI Research Center, University of Minho, Braga, Portugal \\ (sds@dps.uminho.pt; crodrigues@dps.uminho.pt; enunes@dps.uminho.pt )
}

\begin{abstract}
For hospitals, the variability on demand (number of daily urgent admissions) and the variability in the Length of Stay (LoS) (bed occupancy) may affect the quality of service provided to patients and the effectiveness of the overall service. This paper studies the LoS of 238 patients who performed hip surgery in the orthopedic service of a Portuguese hospital in 2014. It uses variables available in electronic databases, such as Age, Gender, ASA classification; Surgical Apgar Score, Type of hip surgery; Weekday of the surgery; Starting hour of the surgery and Duration of surgery to predict LoS and provides a model that correctly indicate if a patient stays more than 7 days in $72.1 \%$ of the cases.
\end{abstract}

Keywords - Bed occupancy, Hip surgery, Hospital, Length of Stay, Quality of service, Statistical analysis

\section{INTRODUCTION}

\section{A. Quality of service in hospitals}

Organizational studies of healthcare are focused on the contribution of organizational solutions to improve performance and healthcare organizations are reflecting on the concept of quality [1].

Quality of service is a multidimensional concept, which involves a comparison of reality with expectations. Examples of Servqual instruments that assess such perceived quality are vast [2]. Two of the five dimensions of Service Quality are: reliability (ability to perform the promised service dependably and accurately) and responsiveness (willingness to help customers and provide prompt service). In the health care sector, in particular in hospitals, the ability to plan admissions and the fulfillment of the required treatments according to expectations of the patient are aligned with these two dimensions. If no beds are available to admit a patient he/she may be rescheduled or admitted in another hospital ward. In both cases it will result in a reduction in the satisfaction associated these dimensions of perceived quality. The hospital may incur additional costs to admit a patient in another location [3].

This work has been supported by COMPETE: POCI-01-0145FEDER-007043 and FCT - Fundação para a Ciência e Tecnologia within the Project Scope: UID/CEC/00319/2013.

Hospital performance can be assessed through a set of performance measures [4], and if no beds are available to allow the admission of new patients that could be reflected in several performance measures such as waiting time for admission, marginal costs and customer satisfaction.

\section{B. Efficiency of use of hospital beds considering unplanned admissions and variable Length of Stay}

Hospitals need to be efficient. Efficiency has several definitions and many authors have referred it relating to different aspects such as costs [5], technical [3] or clinical [6]. As a result of the increase of efficiency it is expected the maximization of the use of medical teams and hospital beds, amongst others.

Considering the use hospital beds, there are two factors that have implications on its management: the variability on demand (number of daily urgent admissions) and the variability in the Length of Stay (LoS) (number of consecutive days a patient occupies a bed). If a patient is not treated due to lack of hospital beds, the patient quality perception for the two above dimensions will decrease [2]. If the hospital needs to put the patient in a different ward may incur with extra costs (moving the care to the patient location and assuring adequate environment) [3], [4].

The LoS could be estimated by the physician at the moment of the admission (or surgery), however, several studies [7], [8] suggest that this may not accurate, nor is recorded and updated for hospital management objectives [9]. This stochastic nature in the admissions and in the LoS makes difficult to use optimization techniques such as Mixed Integer Program or Binary Integer Programs resulting in NP-hard problems and are thus in general challenging [10].

\section{Information available about the LoS}

At the moment of admitting a patient the hospital may create an estimate of the LoS, based on historical data of its diagnosis. The hospital could use general data provided by the Diagnosis-Related Groups [11], it may use hospital databases regarding to similar admissions or it may use more specific information of the patient and associated clinical data, some of which will be updated during its stay.

So, the two factors, bed availability and cost reduction may be in conflict. To have capacity for unplanned admissions, bed capacity should increase but, to reduce operational costs, the total number of beds should be reduced. This can be decided in strategic terms [6], i.e. to define the capacity of a new hospital or change the number 
of beds in an existing hospital, but could also be addressed as an operational problem, i.e. to decide on a daily basis the number of planned admissions based on bed availability forecast [12].

D. Operational decision: which admissions should be planned based on expected bed availability?

In the latter, as an operational problem, the hospital has the possibility of scheduling planned admissions within certain clinical best practices. For example, [10] use a 3 level treatment priority that represents the maximum time for an admission to occur.

\section{E. Objective}

The objective of the study is to examine and study the impact of patients who perform hip surgery in the orthopedic service in a hospital in Northern Portugal. More specifically, it is intended to determine whether the occupancy of the bed after surgery can be predicted by the characteristics of the patient as well as factors specific to surgery.

\section{METHODOLOGY AND SAMPLE}

The study focused on the orthopedic service that has on average 2500 annual admissions, so it is considered the hospital service with the third highest volume of service and one of the highest occupancy rates (about 90\%, hospital information). The orthopedic service is organized into external consultations, ambulatory surgery, operating room surgeries, emergencies and hospitalization. The hospitalization into orthopedic service is exclusively for adults (if less than 18 years, the patient stays in the pediatric service) and has a total of 58 beds organized into two nursing wards by gender of patient.

The database was provided by the hospital and report all surgeries performed during the year 2014. In that year, the orthopedic service held a total of 2,030 surgeries in non-ambulatory. This study specifically analyzes the hip surgeries, since the recovery of these surgeries require greater downtime, then higher hospital bed occupancy time after surgery. In total, there were 269 surgeries to hip (13.3\%). The Length of Stay (LoS) after surgery in hip ranged from 2 to 62 days, with an average value of 9.65 and a standard deviation of 6.727 days (Table I).

TABLE I

HIP SURGERY LENGTH OF STAY CHARACTERIZATION $(\mathrm{N}=269)$

\begin{tabular}{ccccc}
\hline & Min & Max & Average & SD \\
\hline LoS & 2 & 62 & 9,6 & 6,727 \\
\hline
\end{tabular}

The variability of the LoS after surgery is shown in

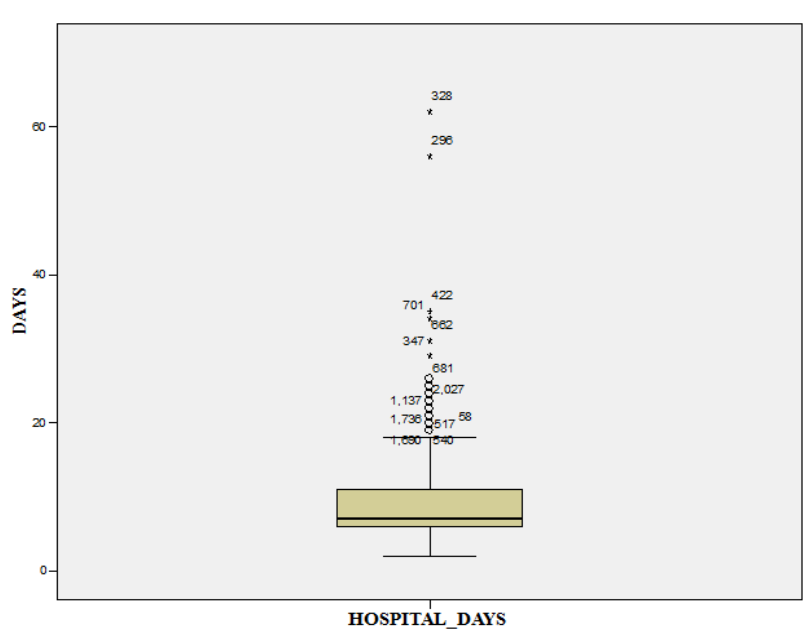

Fig. 1. Boxplot of the distribution of $\mathrm{LoS}$ after hip surgery $(\mathrm{N}=269)$

In terms of age, patients aged from 37 to 97 years, with an average of 70.4 and a standard deviation of 11.477 years. Slightly more than half $(55.8 \%)$ of the hip surgery involved female patients. To quantify the amount of physiological reserve that a patient possesses at the time at which they are assessed for a surgical procedure, the database included the ASA (American Society of Anesthesiologists physical status) of each patient operated to hip. Four levels of ASA were identified (ASA 1 ASA 2 ASA 3 ASA 4), with a predominance of ASA $2(65.4 \%)$ and ASA $3(28.6 \%)$.

\section{DATA ANALYSIS AND RESULTS}

This research intends to examine and predict hospital bed occupancy using characteristics of the patient and factors specific to the surgery. The patient variables included in the study were:

1. Age;

2. Gender;

3. ASA classification;

4. Surgical Apgar Score- measured after surgery (The surgical Apgar score try to predict risk of complication and poor outcome after major surgery. The higher the score, the lower the risk of complication).

The factors specific to the surgery considered in study were:

1. Type of hip surgery;

2. Weekday of the surgery;

3. Starting hour of the surgery;

4. Duration of surgery (hours) - measured after surgery.

Table II and Table III summarize the patient and surgery characterization. Fig.1. 
TABLE II

HIP SURGERY: PATIENT CHARACTERIZATION (N=269)

\begin{tabular}{llccc}
\hline Variable & & $\mathrm{N}$ & $\%$ & \\
\hline Gender & Male & 119 & $44.2 \%$ & \\
& Female & 150 & $55.8 \%$ & \\
\hline ASA & ASA I & 11 & $4.1 \%$ & \\
& ASA II & 176 & $65.4 \%$ & \\
& ASA III & 77 & $28.6 \%$ & \\
& ASA IV & 5 & $1.9 \%$ & \\
\hline Age & Min & Max & Average & SD \\
APGAR & 34 & 97 & 70.4 & 11.477 \\
& 2 & 10 & 7.1 & 1.384 \\
\hline
\end{tabular}

TABLE III

HIP SURGERY: SURGERY CHARACTERIZATION (N=269)

\begin{tabular}{|c|c|c|c|c|}
\hline \multicolumn{3}{|l|}{ Variable } & $\mathrm{N}$ & $\%$ \\
\hline \multirow[t]{4}{*}{ Type } & \multicolumn{2}{|c|}{ Total hip replacement } & 190 & $70.6 \%$ \\
\hline & \multicolumn{2}{|c|}{ Partial hip replacement } & 41 & $15.2 \%$ \\
\hline & \multicolumn{2}{|c|}{ Review of hip replacement } & 28 & $10.4 \%$ \\
\hline & \multicolumn{2}{|c|}{ Another type of hip surgery } & 10 & $3.7 \%$ \\
\hline \multirow[t]{7}{*}{ Weekday } & \multicolumn{2}{|l|}{ Monday } & 36 & $13.4 \%$ \\
\hline & \multicolumn{2}{|l|}{ Tuesday } & 68 & $25.3 \%$ \\
\hline & \multicolumn{2}{|c|}{ Wednesday } & 22 & $8.2 \%$ \\
\hline & \multicolumn{2}{|l|}{ Thurday } & 46 & $17.1 \%$ \\
\hline & \multicolumn{2}{|l|}{ Friday } & 51 & $19.0 \%$ \\
\hline & \multicolumn{2}{|l|}{ Saturday } & 40 & $14.9 \%$ \\
\hline & \multicolumn{2}{|l|}{ Sunday } & 6 & $2.2 \%$ \\
\hline \multirow[t]{5}{*}{ Start hour } & \multicolumn{2}{|c|}{ From $8: 00$ to $11: 50$} & 79 & $29.4 \%$ \\
\hline & \multicolumn{2}{|c|}{ From $12: 00$ to $15: 59$} & 76 & $28.3 \%$ \\
\hline & \multicolumn{2}{|c|}{ From $16: 00$ to $19: 59$} & 61 & $22.7 \%$ \\
\hline & \multicolumn{2}{|c|}{ From 20:00 to $24: 00$} & 53 & $19.7 \%$ \\
\hline & Min & $\operatorname{Max}$ & Average & SD \\
\hline $\begin{array}{l}\text { Duration of } \\
\text { Surgery }\end{array}$ & 0.41 & 4.55 & 1.5 & 0.636 \\
\hline
\end{tabular}

For the analysis, the dependent variable resulted from converting the LoS after surgery into a multinomial variable named involving two categories:

- $\quad$ Superior to median (success)

- $\quad$ Less or equal to median (failure)

The LoS after surgery showed a median of 7 days. Therefore the new variable LoS GREATER 7 DAYS resulted by coding the "yes" answers as 1-yes; otherwise 0no.

To operationalize the categorical variables were performed chi-square tests that allow us to conclude the existence of dependency relationships between the cases of the new variable and the variables:

- Gender $\left[\chi^{2}(1)=11.425, \mathrm{p}<0.01\right]$;

- Surgery type $\left[\chi^{2}(3)=16.376, \mathrm{p}<0.01\right]$;

- Weekday $\left[\chi^{2}(6)=34.504, \mathrm{p}<0.01\right]$ and

- Start hour $\left[\chi^{2}(3)=8.649, \mathrm{p}<0.05\right]$.

Since the interesting variable is a dichotomous variable, a logistic regression analysis was conducted to explore the characteristics of the two cases. Three models have been defined.

The first model used only variables characterizing the patient before surgery (age, gender and ASA), with conversion of the gender categorical variable in a dummy variable (male $=1$, female $=0$ ).

The second model uses only the characterization of surgery before it occurs (surgery type, weekday and start hour), with conversion of categorical variables into dummy variables:

- Hip_revision: review hip replacement $=1$, other $=0$;

- Weekday_wednesday: wednesday $=1$, other $=0$;

- Twelve to fifteen: yes $=1$, other $=0$

The third model considers the after surgery and uses all the available variables, including the patient's APGAR (measured after surgery) and duration of surgery.

The results are summarized in Table IV.

In Model 1, the MALE and ASA variables significantly contribute to the prediction (both with a pvalue $<0.01$ ) but the variable AGE has no explanatory power. The negative sign of the dummy MALE suggests that in the case of male patients LoS is shorter than the female patients, which is reinforced by $\operatorname{EXP}(B)$ less than 1 (in the case of male patients, the value of $\operatorname{EXP}(B)=0.440$ means that the female patients are $1 / 0.440=2.273$ times more likely to belong to the group of which remain over 7 days in the hospital.

In Model 2, the three explanatory variables contribute to the prediction (HIP_REVISION and WEEKDAY_WEDNESDAY both with p-value $<0.01$, in the case of start hour TWELVE TO FIFTEEN with a p-value $<0.10$ ). The logistic coefficients can be used to create a predictive equation. In our study, the Model 2 resulted in the following equation: 
TABLE IV

REGRESSION LOGISTICS COEFICIENTS

\begin{tabular}{|c|c|c|c|c|c|c|c|c|c|}
\hline \multirow{2}{*}{ Variables } & \multicolumn{3}{|c|}{ Model 1} & \multicolumn{3}{|c|}{ Model 2} & \multicolumn{3}{|c|}{ Model 3} \\
\hline & Coefficient & SIG & $\operatorname{EXP}(B)$ & Coefficient & SIG & $\operatorname{EXP}(B)$ & Coefficient & SIG & $\mathrm{EXP}(\mathrm{B})$ \\
\hline INTERCEPT & -0.538 & 0.549 & 0.584 & -0.600 & 0.000 & 0.549 & -2.770 & 0.062 & 0.063 \\
\hline AGE & -0.019 & 0.149 & 0.981 & - & - & - & -0.011 & 0.441 & 0.989 \\
\hline MALE & -0.821 & 0.003 & 0.440 & - & - & - & -0.833 & 0.005 & 0.435 \\
\hline ASA & 0.912 & 0.001 & 2.488 & - & - & - & 0.964 & 0.001 & 2.623 \\
\hline APGAR & - & - & - & - & - & - & 0.072 & 0.481 & 1.074 \\
\hline HIP_REVISION & - & - & - & 1.812 & 0.000 & 6.124 & 1.694 & 0.005 & 5.439 \\
\hline WEEKDAY_WEDNESDAY & - & - & - & 2.183 & 0.001 & 8.869 & 1.967 & 0.003 & 7.152 \\
\hline TWELVE TO FIFTEEN & - & - & - & 0.564 & 0.055 & 1.757 & 0.522 & 0.091 & 1.686 \\
\hline DURATION & - & - & - & - & - & - & 0.425 & 0.136 & 1.530 \\
\hline CHI-SQUARE & \multicolumn{3}{|c|}{24.200} & \multicolumn{3}{|c|}{37.659} & \multicolumn{3}{|c|}{62.801} \\
\hline COX \& SNELL R SQUARE & \multicolumn{3}{|c|}{0.086} & \multicolumn{3}{|c|}{0.131} & \multicolumn{3}{|c|}{0.208} \\
\hline NAGELKERKE R SQUARE & \multicolumn{3}{|c|}{0.115} & \multicolumn{3}{|c|}{0.174} & \multicolumn{3}{|c|}{0.278} \\
\hline -2 LOG LIKELIHOOD & \multicolumn{3}{|c|}{347.638} & \multicolumn{3}{|c|}{334.179} & \multicolumn{3}{|c|}{309.037} \\
\hline $\begin{array}{l}\text { CLASSIFYING OVERALL } \\
\text { PERCENTAGE }(\%)\end{array}$ & \multicolumn{3}{|c|}{$60.2 \%$} & \multicolumn{3}{|c|}{$65.1 \%$} & \multicolumn{3}{|c|}{$72.1 \%$} \\
\hline
\end{tabular}

$p=\frac{\exp \{-0.600+1.812 \times H R+2.183 \times W W+0.564 \times T F\}}{1+\exp \{-0.600+1.812 \times H R+2.183 \times W W+0.564 \times T F\}}$

Where:

$\mathrm{p}=$ probability that a case belong to the category "LoS greater than 7 days";

$\mathrm{HR}=$ Hip revision;

$\mathrm{WW}=$ Weekday_Wednesday (surgery schedule to a Wednesday) and;

$\mathrm{TF}=$ twelve to fifteen (surgery schedule to start from $12: 00$ to $15: 59)$.

As an example, imagine a surgery scheduled to start by 13:00 on a Wednesday. If the surgery is a review of hip replacement, the probability that surgery will result in a LoS greater than 7 days is $98.1 \%$. If the surgery is reschedule for a day other than Wednesday (for instance Thursday or Monday), the probability decreases to $85.5 \%$.

In Model 3, it is confirmed the explanatory power of variables MALE, ASA, HIP_REVISION, WEEKDAY_WEDNESDAY (all with a p-value $<0.01$ ) and TWELVE TO FIFTEEN (with a p-value $<0.10$ ). In turn, the AGE variable, APGAR and DURATION of surgery have no explanatory power. Nevertheless, the combination in Model 3 of the patient's characteristics and the characteristics of the surgery allows a classification results with an overall percentage of $72.1 \%$ of cases correctly assigned to the two categories considered in the response variable.

\section{IV - DISCUSSION AND CONCLUSION}

The aim of the research was to study the impact on the LoS of the hip surgery patients in the orthopedic ward of a hospital in northern Portugal. Using information provided by the hospital on 238 patients subjected to hip surgery, it analyzed the bed occupancy after surgery in one of two categories classification: LoS higher than 7 days (median duration); LoS less than or equal to 7 days. The models developed in logistic regression used as explanatory variables patient characteristics as well as specific factors of the surgery to hip. The first model considered only the characteristics of the patient before surgery (age, gender and ASA). The second model used only surgery characteristics prior to its occurrence (type of surgery, day schedule and time of surgery). The third and final model used all the available variables before and after surgery, including the patient Apgar and duration of the surgery. The third model resulted in a correct classification of $72.1 \%$ of cases.

The analysis of the models has highlighted the explanatory power of the variables gender (male), ASA, type of surgery (revision to the hip) and schedule of the day (Wednesday). Although limited in view of the information gathered and provided by the hospital, these results offer an interesting basis for analysis of the factors to consider in the timing of the hip surgery and its impact on LoS and consequent quality of the hospital's orthopedic service. In addition to the implications for the patient's expectations subject to surgery, a stay lasting longer has a direct effect on the availability of beds, and into the management of the planned surgeries. 
This work is integrated in a bigger project aiming to develop a methodology, that estimates in a given day $t$, the number of available beds in day $t+n$, considering existing information available in hospital databases about patients (general information and clinical data).

The results obtained in the initial stage of research indicate the need for more careful and systematic information gathering by the hospital. Although the hospital has provided the ASA classification of each patient, it was not possible to consider, in the tested models, more detailed information about the health status of the patient (e.g. the existence of any debilitating health conditions such as diabetes, hypertension or overweight, among others). Moreover, in the specific factors of the hip surgery, it was not possible to identify the surgical teams and thus to study their impact on the LoS of the patient.

During the next stages of research, efforts will be made with the hospital management in order to address these situations. It is also planned to extend the current research to other Portuguese hospitals and other surgical services.

\section{REFERENCES}

[1] M. Giannini, "Performance and quality improvement in healthcare organizations," International Journal of Healthcare Management, vol. 8, no. 3, pp. 173-179, 2015.

[2] A. Parasuraman, L. L. Berry, and V. Zeithaml, "Refinement and Reassessment of the SERVQUAL Scale," Journal of Retailing, vol. 67, no. 4, pp. 420-450, Winter 1991.

[3] J. M. Sutherland, "Pricing hospital care: Global budgets and marginal pricing strategies," Health Policy, vol. 119, no. 8, pp. 1111-1118, August 2015.

[4] P. J. Van der Wees, M. W. G. Nijhuis-van der Sanden, E. van Ginneken, J. Z. Ayanian, E. C. Schneider, and G. P. Westert, "Governing health care through performance measurement in Massachusetts and the Netherland," Health Policy, vol. 116, no. 1, pp. 18-26, May 2014.

[5] F. Eijkenaar, M. Emmert, M. Scheppach, and O. Schöffski, "Effects of pay for performance in health care: A systematic review of systematic reviews," Health Policy, vol. 110, no. 2-3, pp. 115-130, May 2013.

[6] L. Seematter-Bagnoud, S. Fustinoni, D. H. Dung, B. SantosEggimann, V. Koehn, R. Bize, A. Oettli, and J.-B. Wasserfallen, "Comparison of different methods to forecast hospital bed needs," European Geriatric Medicine, vol. 6, no. 3, pp. 262-266, 2015.

[7] J. Jakubaschk, D. Waidvogel, and O. Wiirmle, "Differences between long-stay and short-stay inpatients and estimation of length of stay A prospective study," Soc Psychiatry Psychiatr Epidemiol, vol. 28, no. 2, pp. 84-90, Apr. 1993.

[8] P. Nassar Jr, and P. Caruso, "ICU physicians are unable to accurately predict length of stay at admission: a prospective study," International Journal for Quality in Health Care, vol. 28, no. 1, pp. 99-103, 2016.

[9] J. K Cochran, and K Roche, "A queuing-based decision support methodology to estimate hospital inpatient bed demand," Journal of the Operational Research Society, vol. 59, no. 11, pp. 1471-1482, Nov. 2008.

[10] R. Scmidt, S. Geisler, and C. Spreckelsen, "Decision support for hospital bed management using adaptable individual length of stay estimations and shared resources," $B M C$
Medical Informatics \& Decision Making, vol. 13, no. 3, pp. 1-19, 2013.

[11] B. Reinhard, A. Geissler, W. Quentin, and M. Wilm (Eds), Diagnosis-Related Groups In Europe: Moving towards transparency, efficiency and quality in hospitals, McGrawHill/Open University Press, UK, 2011.

[12] J. M. Nguyen, P. Six, D. Antonio, P. Glemain, G. Potel, P. Lombrail, and P. Le Beux, "A simple method to optimize hospital beds capacity," International Journal of Medical Informatics, vol. 74, no. 1, pp. 39-49, Jan. 2005. 